\title{
Tolosa-Hunt syndrome masquerading as a carotid artery dissection
}

\author{
This article was published in the following Dove Press journal: \\ Clinical Ophthalmology \\ 07 April 2014 \\ Number of times this article has been viewed
}

\author{
Elise J Taylor' \\ Ursula M Anders' \\ Joseph R Martel ${ }^{1-4}$ \\ James B Martel ${ }^{1-4}$ \\ 'Research Center, Martel Eye Medical \\ Group, Rancho Cordova, ${ }^{2}$ Graduate \\ Medical Education, California \\ Northstate University College \\ of Medicine, Elk Grove, ${ }^{3}$ Department \\ of Ophthalmology, Sutter Medical \\ Health, Sacramento, ${ }^{4}$ Department \\ of Ophthalmology, Dignity Health, \\ Carmichael, CA, USA
}

Purpose: To demonstrate the difficulties of diagnosing a patient with Tolosa-Hunt syndrome (THS) due to its complicated presentation and extensive diagnostic testing, and how to manage the treatment of a patient in an emergent setting.

Patients and methods: A female patient with THS affecting the left eye was examined using two magnetic resonance imaging (MRI) scans. The patient was treated with high-dose methylprednisolone (Solu-Medrol ${ }^{\mathbb{}}$ ) and prednisone. A follow-up MRI and magnetic resonance angiogram (MRA) was also performed 4 months later.

Results: The second MRI scan disclosed a $5 \times 9 \times 10 \mathrm{~mm}$ lesion in the left superior orbital fissure/cavernous sinus. After administration of methylprednisolone and prednisone, the patient's pain completely resolved, and the left eye regained full duction and eyelid mobility. The MRI and MRA obtained after the treatment showed no abnormalities.

Conclusion: The rarity of THS makes it difficult to diagnose, especially when there is a question of accuracy and reproducibility of the testing performed. An ophthalmologic consultation in such cases is crucial.

Keywords: granulomatous lesion, painful ophthalmoplegia, idiopathic orbital inflammation, multiple cranial nerve palsies

\section{Introduction}

Painful ophthalmoplegia (PO) is a common presentation of a dissection of the internal carotid artery (ICA), and is believed to be caused by a disruption of blood flow to the vascular supply of the inferolateral trunk. ${ }^{1}$ It can be associated with headaches or facial pain, and may involve other cranial nerves. ${ }^{1-3}$ The differential diagnosis for PO includes aneurysms, thrombosis, parasellar lesions, tumors, orbital pseudotumor, sarcoidosis, infection, head and neck trauma, and hypertensive or diabetic microvascular infarction. ${ }^{2,4}$ The clinical presentation is also similar to Tolosa-Hunt syndrome (THS), which is a disease that results from a cryptogenic granulomatous lesion within the cavernous sinus/superior oblique fissure of the orbit. It produces PO with ipsilateral periorbital or hemicranial pain. ${ }^{2,4-6}$ The myriad of presentations and rarity of this condition continues to make THS an elusive diagnosis.

\section{Case report}

A 58 year-old female was evaluated for chest pain, bradycardia, severe headache, and left periorbital pain with ipsilateral ophthalmoplegia. A warm sensation on the left side of her face with decreased sensation to light touch was noted. Her past medical history included hypertension, arrhythmia, and trauma. She previously visited the emergency department at Sutter Memorial Hospital (Sacramento, CA, USA) on two separate occasions for severe hemicranial pain, which remained constant for 1 week. Computed
Correspondence: James B Martel California Northstate University College of Medicine, 9700 West Taron Drive, Elk Grove, CA 95757, USA

Tel + I 9166867300

Fax +19166867310

Email james.martel@cnucom.org 


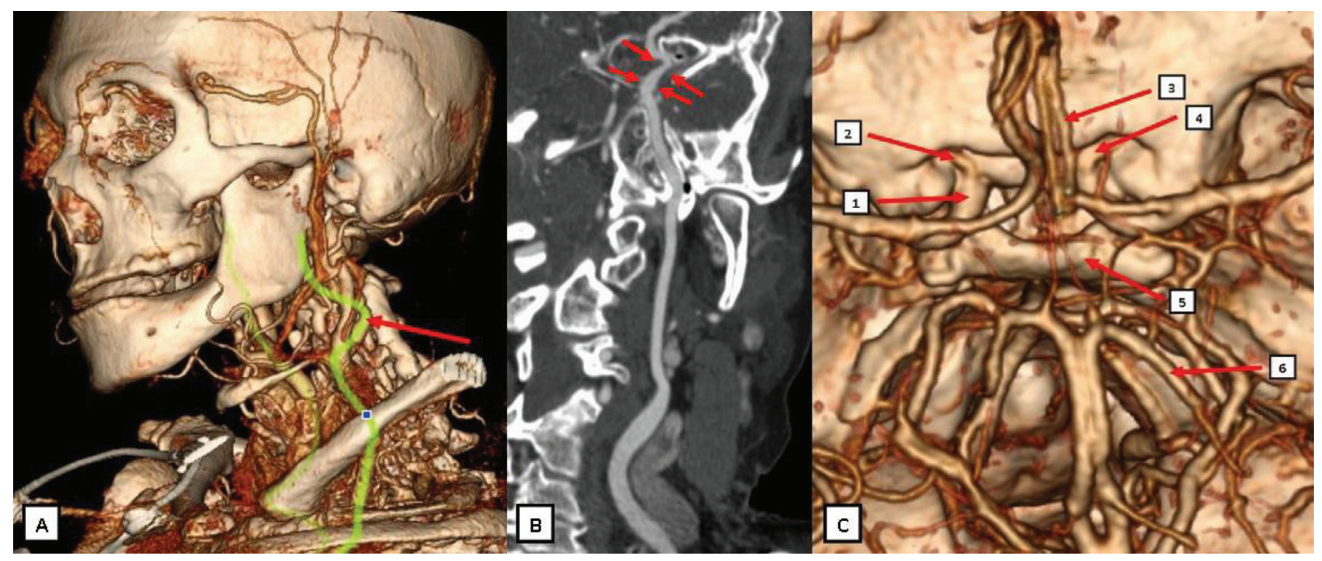

Figure I Three-dimensional reconstructed view of the CT angiogram.

Notes: Three-dimensional reconstructed view of the CT angiogram displaying the left internal carotid artery (red arrow) (A), followed by a two-dimensional perspective displaying $39 \%$ stenosis of the intracavernous portion of the internal carotid artery (red arrow) (B). (C) Three-dimensional view displaying the internal carotid artery at the level of the Circle of Willis: I internal carotid artery, 2 ophthalmic artery, 3 anterior cerebral artery, 4 optic nerve, 5 anterior lobe of pituitary gland, 6 posterior cerebral artery.

Abbreviation: CT, computed tomography.

tomography (CT) scans of the brain without contrast were performed during both visits, with neither showing any acute intracranial abnormalities. CT angiogram (CTA) showed no signs of a dissecting ICA or mass in the superior stellate ganglion of the neck, but it revealed $39 \%$ stenosis of the left ICA at the intracavernous portion (Figure 1). No enlargement of the superior orbital vein was noted. The patient was observed to be bradycardic with a pulse ranging from 29 to 50 beats per minute, including a 3.2 second pause. However, electrocardiogram, cardiac serology (creatinine kinase, creatinine kinase-muscle/brain enzyme type, troponin I, prothrombin time, partial thromboplastin time), chest radiography, and coronary arteriography were all normal. Initial magnetic resonance imaging (MRI) and magnetic resonance angiogram (MRA) showed no abnormalities of the orbital apex or surrounding structures. Thrombus was not observed in the superior orbital vein or cavernous sinus. Neurologic consultation considered it to be an isolated third nerve palsy.

Ophthalmologic evaluation disclosed complete ptosis and ophthalmoplegia in the left eye, with the eye in central position (Figure 2). Proptosis was not noted. Visual acuity was 20/25 in the right eye and 20/50 in the left eye. Both pupils were equal, round, and reactive to light. Intraocular pressures were normal. Ophthalmoscopy revealed a slightly edematous left optic nerve, but no engorgement or tortuosity of the retinal vasculature. Ophthalmologic findings were consistent with a partial third, and complete fourth and sixth nerve paresis with hypoesthesia in the ophthalmic and maxillary distributions of the left trigeminal nerve. A subsequent MRI performed 3 days after the initial MRI disclosed a lesion in the left superior orbital fissure with

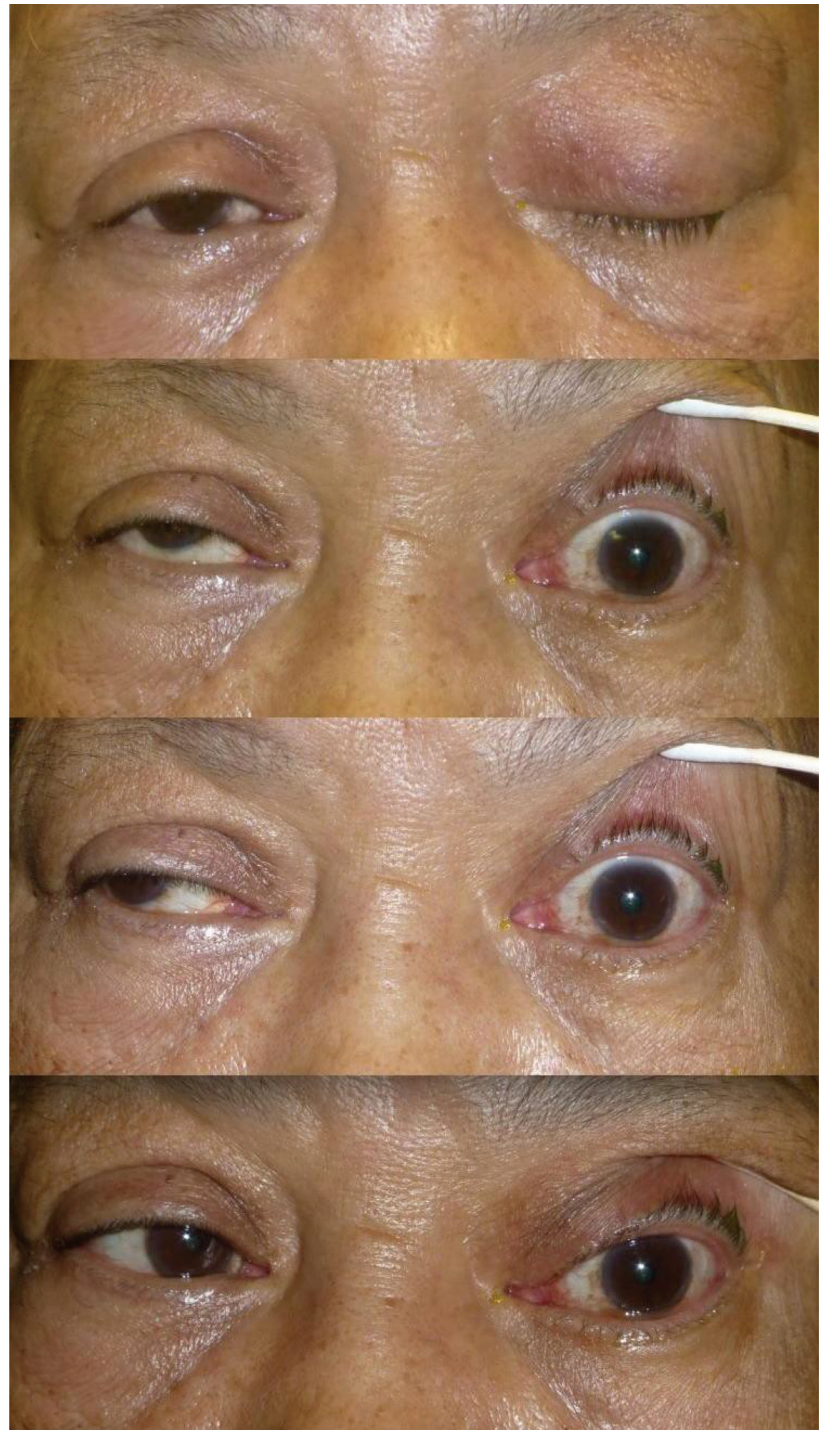

Figure 2 Complete ptosis and ophthalmoplegia in the left eye. 


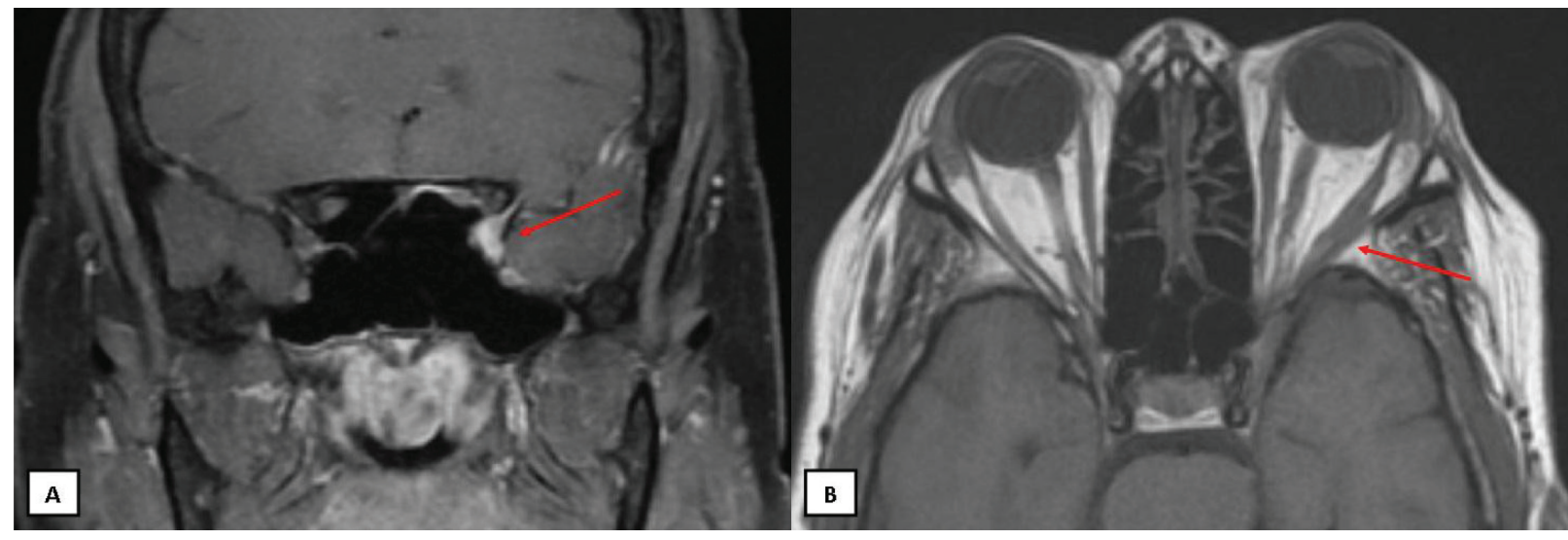

Figure $3 \mathrm{TI}$ and T2-weighted MRI with gadolinium contrast of the brain and orbits.

Notes: Coronal T2-weighted MRI disclosing a $5 \times 9 \times 10 \mathrm{~mm}$ abnormal enhancing of the soft tissue in the left superior orbital fissure with mild extension along the anterior aspect of the left cavernous sinus (red arrow) (A), and an axial TI-weighted MRI disclosing mild edema of the left lateral rectus (red arrow) (B).

Abbreviations: MRI, magnetic resonance imaging. TI, spin-lattice relaxation time; T2, spin-spin relaxation time.

mild extension along the anterior aspect of the left cavernous sinus (Figure 3A), and mild edema of the lateral rectus (Figure 3B). Complete blood count, thyroid stimulating hormone, glucose, sedimentation rate, rheumatoid factor, antinuclear antigen, proteinase 3 antibodies, myeloperoxidase antibodies, Quantiferon ${ }^{\circledR}$-TB Gold (Cellestis Limited, Carnegie, VIC, Australia), human immunodeficiency virus, herpes simplex virus, and fluorescent treponemal antibody absorption test results were normal. Two complete sets of cerebrospinal fluid studies (CSF), including opening pressure, India ink smear, glucose, total protein, angiotensin converting enzyme, and a venereal disease research laboratory test showed no evidence of malignancy or infection. The patient was administered $1 \mathrm{~g}$ of intravenous (IV) methylprednisolone (Solu-Medrol ${ }^{\circledR}$; Pfizer, New York, NY, USA) over 45 minutes, followed by $250 \mathrm{mg}$ every 8 hours daily for 3 days while in hospital. She was discharged with $40 \mathrm{mg}$ of oral prednisone on a tapered course ( $40 \mathrm{mg}$ for 5 days, $20 \mathrm{mg}$ for 5 days, $10 \mathrm{mg}$ for 5 days, $5 \mathrm{mg}$ for 5 days, $2.5 \mathrm{mg}$ for 5 days, then discontinued).

The patient's periorbital and hemicranial pain was reduced within 48 hours. She was referred for neurosurgical consultation, but she declined. An additional MRI/MRA was obtained at a 4-month follow-up and revealed no lesions or abnormalities. The ophthalmoplegia and ptosis had also resolved. She continues to be monitored and remains symptom free (March 2014).

Written informed consent was obtained from the patient to allow the publication of photographs for educational purposes. This study adhered to the tenets of the Declaration of Helsinki and was Health Insurance Portability and Accountability Act (HIPAA) compliant. Sutter Health Central Area
Institutional Review Committee ruled that approval was not required for this study.

\section{Discussion}

When evaluating a patient with PO, an expeditious systematic approach should be utilized to rule out any emergent etiologies. Vascular origins such as dissection of the ICA, aneurysm, and cavernous sinus thrombosis require immediate attention. ${ }^{2}$ CTA and MRI are the standard measures for diagnosing vascular causes of ophthalmoplegia. The sensitivity of a CTA ranges from $98 \%$ to $100 \%$ in detecting aneurysms less than $5 \mathrm{~mm}$ in diameter, ${ }^{7}$ and the sensitivity of MRI combined with MRA approaches $100 \%$ in detecting cerebral vein thrombosis. ${ }^{8}$ Furthermore, ophthalmoscopy can detect venous dilation and tortuosity in the setting of an occluded superior ophthalmic vein, as would occur with a carotid cavernous thrombosis. In the presence of normal imaging and laboratory studies, hypertensive microvascular infarction can be considered as the cause of $\mathrm{PO},{ }^{2,9}$ but hypertension alone has not been shown to cause paralysis of the third, fourth, fifth, and sixth cranial nerves simultaneously. ${ }^{9-11}$

One of the rare causes of PO is THS, which results from a granulomatous inflammation in the region of the superior orbital fissure/cavernous sinus. ${ }^{2,4-6}$ In the past it has been a condition of exclusion. We describe a hypertensive patient who presented with $\mathrm{PO}$, with a mild narrowing of the intracavernous portion of the ICA, and normal laboratory test results. Stenosis of the ICA was found by CTA, which is commonly observed with THS, ${ }^{2,4}$ but the diagnosis could not be validated with a negative MRI. A repeat MRI very clearly showed a lesion in the superior oblique fissure, indicating THS. The lesion was not present on the initial 
MRI, which can be explained by disease evolution or volume averaging (slice thickness). No one specific study has addressed this complexity, and some have suggested to proceed with treatment and to determine whether or not the patient has THS based on their response to steroids. ${ }^{12,13}$ The clinical and imaging response to high-dose steroids has been considered to be one of the principal criteria of diagnosing THS. ${ }^{4,5}$ Infectious causes of this or any other syndrome are exacerbated by using high-dose steroids, and microvascular causes may exhibit worsening. Neoplasms may initially respond to steroids, which would provide a false sense of security. Thus, treatment with high-dose steroids may be an issue of controversy. We would recommend a repeat MRI study when serologic and CSF studies are normal and the clinical suspicion for THS remains.

THS may be part of the same pathological process of idiopathic orbital inflammatory disease, confined to the cavernous sinus/superior orbital fissure. ${ }^{14}$ In this case report, the presence of an enlarged ipsilateral lateral rectus muscle and optic nerve edema with visual loss would support this etiology. We emphasize the fact that patients with this diagnosis should be followed carefully as neoplasms can have "false positive" responses to steroids, ${ }^{4}$ and a full understanding of this condition has not yet been obtained.

\section{Acknowledgment}

We would like to thank Michelle Hoyt and Victoria Kravchuk for their data acquisition and drafting contributions that led to the completion of this article.

\section{Disclosure}

The authors report no conflicts of interest in this work.

\section{References}

1. Vargas ME, Desrouleaux JR, Kupersmith MJ. Ophthalmoplegia as a presenting manifestation of internal carotid artery dissection. $J$ Clin Neuroophthalmol. 1992;12(4):268-271.

2. Gladstone JP, Dodick DW. Painful ophthalmoplegia: overview with a focus on Tolosa-Hunt syndrome. Curr Pain Headache Rep. 2004; 8(4):321-329.

3. Mokri B, Silbert PL, Schievink WI, Piepgras DG. Cranial nerve palsy in spontaneous dissection of the extracranial internal carotid artery. Neurology. 1996;46(2):356-359.

4. Kline LB, Hoyt WF. The Tolosa-Hunt syndrome. J Neurol Neurosurg Psychiatry. 2001;71(5):577-582.

5. Hunt WE. Tolosa-Hunt syndrome: one cause of painful ophthalmoplegia. J Neurosurg. 1976;44:544-549.

6. Odabasi Z, Gokcil Z, Atilla S, Pabuscu Y, Vural O, Yardim M. The value of MRI in a case of Tolosa-Hunt syndrome. Clin Neurol Neurosurg. 1997;99(2):151-154.

7. Villablanca JP, Jahan R, Hooshi P, et al. Detection and characterization of very small cerebral aneurysms by using $2 \mathrm{D}$ and $3 \mathrm{D}$ helical CT angiography. AJNR Am J Neuroradiol. 2002;23(7):1187-1198.

8. Lafitte F, Boukobza M, Guichard JP, et al. MRI and MRA for diagnosis and follow-up of cerebral vein thrombosis (CVT). Clin Radiol. 1997;52(9):672-679.

9. Rush JA, Younge BR. Paralysis of cranial nerves III, IV, and VI. Cause and prognosis in 1,000 cases. Arch Ophthalmol. 1981;99(1):76-79.

10. Berlit P. Isolated and combined pareses of cranial nerves III, IV, and VI. A retrospective study of 412 patients. J Neurol Sci. 1991;103(1):10-15.

11. Park UC, Kim SJ, Hwang JM, Yu YS. Clinical features and natural history of acquired third, fourth, and sixth cranial nerve palsy. Eye (Lond). 2008;22(5):691-696.

12. Aktan S, Aykut C, Erzen C. Computed tomography and magnetic resonance imaging in three patients with Tolosa-Hunt syndrome. Eur Neurol. 1993;33(5):393-396.

13. Smith JL, Taxdal DSR. Painful ophthalmoplegia. The Tolosa-Hunt syndrome. Am J Ophthalmol. 1966;61(6):1466-1472.

14. Wasmeier C, Pfadenhauer K, Rosler A. Idiopathic inflammatory pseudotumor of the orbit and Tolosa-Hunt syndrome - are they the same disease? J Neurol. 2002;249(9):1237-1241.
Clinical Ophthalmology

\section{Publish your work in this journal}

Clinical Ophthalmology is an international, peer-reviewed journal covering all subspecialties within ophthalmology. Key topics include: Optometry; Visual science; Pharmacology and drug therapy in eye diseases; Basic Sciences; Primary and Secondary eye care; Patient Safety and Quality of Care Improvements. This journal is indexed on Submit your manuscript here: http://www.dovepress.com/clinical-ophthalmology-journal

\section{Dovepress}

PubMed Central and CAS, and is the official journal of The Society of Clinical Ophthalmology (SCO). The manuscript management system is completely online and includes a very quick and fair peer-review system, which is all easy to use. Visit http://www.dovepress.com/ testimonials.php to read real quotes from published authors. 